\title{
Respiration rate detection based on intensity modulation using plastic optical fiber
}

\author{
Zawawi Mohd Anwar ${ }^{1, *}$, Nor Ziran Nurul Sufia ${ }^{1}$, and Manap $\mathrm{Hadi}^{2}$ \\ ${ }^{1}$ Faculty of Electrical \& Electronics Engineering, Universiti Malaysia Pahang, 26600 Pekan, Malaysia \\ ${ }^{2}$ Faculty of Engineering Technology, Universiti Malaysia Pahang, 26600 Pekan, Malaysia
}

\begin{abstract}
This paper presents the implementation of respiration rate measurement via a simple intensitybased optical fiber sensor using optical fiber technology. The breathing rate is measured based on the light intensity variation due to the longitudinal gap changes between two separated fibers. In order to monitor the breathing rate continuously, the output from the photodetector conditioning circuit is connected to a low-cost Arduino kit. At the sensing point, two optical fiber cables are positioned in series with a small gap and fitted inside a transparent plastic tube. To ensure smooth movement of the fiber during inhale and exhale processes as well as to maintain the gap of the fiber during idle condition, the fiber is attached firmly to a stretchable bandage. This study shows that this simple fiber arrangement can be applied to detect respiration activity which might be critical for patient monitoring.
\end{abstract}

\section{Introduction}

This paper presents the development of a simple and low cost respiration sensor based on intensity modulation technique using fiber displacement loss between two separated plastic optical fibers. Two plastic fibers are applied; the one connected to the light source is the emitting fiber and the other fiber attached to the photodetector is the receiving fiber. The objective of this study is to determine the potential sensor arrangement using plastic optical fiber that can be applied to detect the respiration rate from the chest movement.

A brief review on currently available techniques for measuring respiration process using optical and nonoptical devices is discussed in brief. This is followed by a description of the sensor configuration presented in this paper. Related theoretical information is also presented to describe the fiber displacement effect on the light intensity.

\section{Review on current optical fiber sensor for respiration measurement}

The development of various sensor approaches for respiration measurement are available in many research articles. Some popular devices for this particular application are capacitive sensor [1], low-power radar system [2], vision system [3] and optical fiber technique using temperature sensor at nostril [4] and macro-bend sensor embedded into a stretchable textile [5].

For the first type of respiration detection using capacitive sensor [1], two capacitive sensors were designed and fabricated for detecting chest or abdominal circumference. The sensor is placed on a respiration belt and can be applied to detect chest variation if maximum $60 \mathrm{~mm}$. In theory, capacitive sensors work by measuring changes in an electrical property called capacitance, commonly with two conductive objects with a space between them respond to a voltage difference applied to them. As the respiration process takes place, the changes in capacitance value are monitored, which in this application representing the changes in chest circumference.

For the second device type [2], the respiration is detected alongside with the heartbeat using a $5 \mathrm{GHz}$ radar system. This technique applied the concept of Doppler's effect to detect the chest movement during breathing without any physical contact with the human body. As a result, subject under study can be allowed to move freely inside a controlled room during the monitoring process without changing their normal respiration activity. However, this technique suffers from the harmonics effect of the respiration signal which may interfere with the detection of heartbeat rate and thus reduce its accuracy.

Another interesting research is based on the video image system [3]. In this study, the image and signal processing techniques were used to extract the chest and abdominal movement's information from a series of recorded video using a single camera. The data was stored for further offline analysis to determine the respiration rate based on the time sequence of the recorded video. As applied for other detection application using camera system, this technique is rather expensive for normal health monitoring needs. Moreover, frequent calibration process is important to guarantee the accuracy of the

\footnotetext{
* Corresponding author: mohdanwar@ump.edu.my
} 
obtained measurement results, which could be timeconsuming.

As far as optical fiber technique is concerned, the respiration monitoring can be implemented via the heat detection at the nostril and/or the chest movement. The first technique is realized using an optical temperature sensor placed at the nostril [4]. A sensing material called thermo-chromic microcapsule pigment was selected as the detection surface because this material changes its colour from red to white upon the absorption of heat (during exhale cycle). The intensity of the reflected light was monitored at the output which was corresponding to the changes in the pigment film colour. Second optical sensor technique is based on the macro-bend technique [5] using optical fiber cable. In this case, the optical fiber was stitched on a flexible garment taking the "U-shape" form. The elasticity of the garment allows the attached fiber to experience changes in the curve radius, thus changes the intensity of the detected light. As the breathing process take place, the light intensity also varies according to the chest expansion.

In this paper, a rather similar fiber optic material is used but the light intensity variation is altered due to fiber displacement gap changes that represent the chest movement during respiration process. In order to identify the suitable initial gap for the fiber installation on the stretchable bandage, several experiments have been conducted. Finally, the assembled optical sensor is connected to suitable LED driver and signal conditioning circuits before the output of the circuit is displayed on the monitor screen via Arduino kit interface.

\section{Theory on fiber displacement gap effect on light intensity}

Many previous studies have presented the relationship between the light intensity of a plastic fiber due to different fiber movement; namely longitudinal gap, lateral offset, tilting angle offset and reflective gap between fiber end tip and a reflective mirror [6]. For the explanation on the optical sensor implemented in this study, only the effect of longitudinal gap between the emitting and receiving fibers is further discussed. Particularly for this fiber movement, the light intensity at the receiving fiber is dependent on multiple parameters such as refractive index of the medium at the fibre gap $(\mathrm{nm})$, refractive index of the applied fiber core $\left(n_{c}\right)$ and cladding $\left(n_{c l}\right)$, radius of the fibre core (a) as well as the separation distance the sensor gap (z).

Figure 1 illustrates this relationship which shows the main parameters involved in the theoretical estimation. The equation is obtained from preliminary study by Tsuchiya et. al. [7] :

$$
\frac{P_{o}}{P_{i}}=\frac{16 K^{2}}{(1+K)^{4}} \cdot\left(1-\frac{z}{4 a} \cdot K \cdot(2 \cdot \Delta)^{0.5}\right)
$$

Where:

$$
K=\frac{n_{c}}{n_{m}}
$$

Po - the output power at the receiving fibre due to displacement.

$P i$ - the output power at the receiving fibre at zero gap.

$n_{c}$ - the refractive index of the fibre core.

$n_{c l}$ - the refractive index of the fibre cladding.

$n_{m}$ - the refractive index of the medium between the fibres

$z$ - the longitudinal gap between input and output fibre.

$a$ - the fibre core radius.

$\Delta$ - the refractive index difference between core and cladding $\left(\mathrm{n}_{\mathrm{c}}-\mathrm{n}_{\mathrm{cl}}\right)$

For the applied fiber type in this study (Mitsubishi Rayon simplex POF), the numerical aperture, NA $=0.5$, fiber core radius, $a=0.49 \mathrm{~mm}$, core refractive index, $n_{c}=$ 1.492 , cladding refractive index, $n_{c l}=1.402$, and the refractive index difference, $\Delta=0.09$. These results in the calculated value of $\mathrm{K}=1.0642$. As the emitting and receiving fibers are connected together in air, which refractive index, $\mathrm{nm}=1.000293 \cong 1.00$, the normalized output intensity estimation versus longitudinal displacement using Eq. 1 above is illustrated in Figure 2.

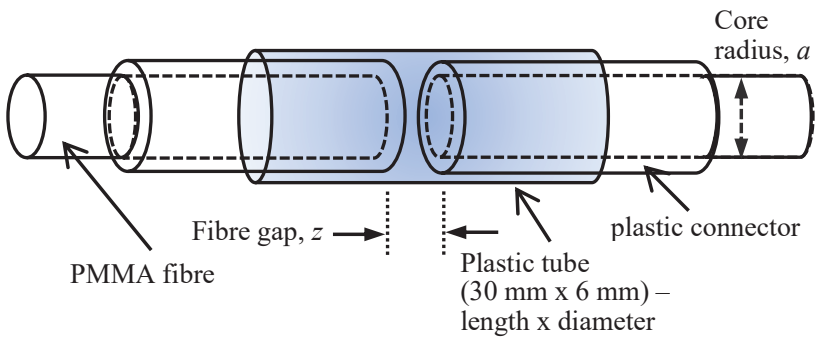

Fig. 1. Side-polish optical fiber bending sensor.

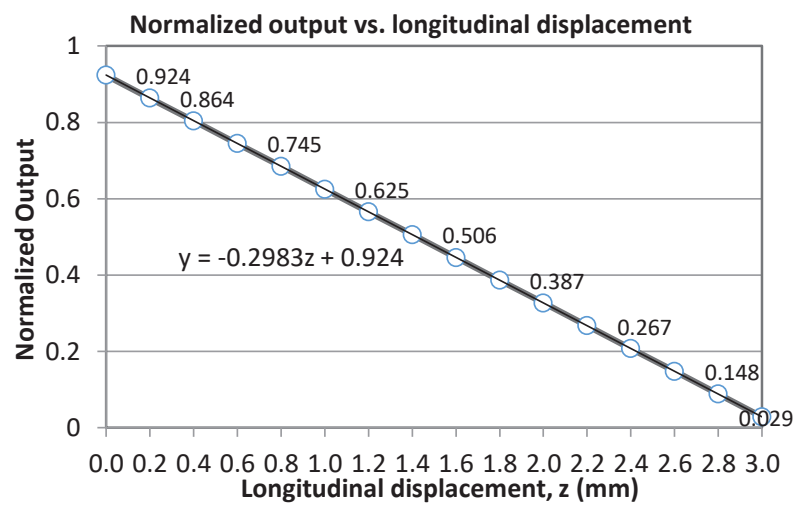

Fig. 2. Relationship between the fiber gap and the output intensity.

From the relationship between the normalized output and the longitudinal displacement of the emitting and receiving fiber end tips as shown in Figure 2, it was observed that a linear relationship is obtained for a displacement gap between $0.0 \mathrm{~mm}$ to $3.0 \mathrm{~mm}$. Additional fiber gap will result in poor light intensity at the receiving fiber, thus should be avoided in the sensor development. 


\section{Sensor configuration of this investigation}

As previously mentioned, fiber gap displacement technique based on intensity-modulation between the emitting and receiving fibers has been implemented as a respiration sensor aimed to monitor the chest expansion during inhalation and exhalation processes. In terms of the sensor arrangement, it is important to have a consistent output readings for a certain period of time (for continuous monitoring purpose), so that the light intensity variation from the optical sensor is only due to the chest diameter variation. Other associated factors, such as variation in light intensity brightness, photodetector sensitivity and supply voltage fluctuation could lead to sensor measurement inaccuracy. In this study, we only focused on the sensor assembly on a stretchable material (i.e. standard clinical bandage) to ensure a firm sensor attachment on its surface and basic laboratory tests to verify the sensor arrangement suitability for the aimed application.

In order to have a sufficient light reception at the receiving fiber before being attached on the bandage, the fiber gap (end tip distance between emitting and receiving fibers) of less than $3 \mathrm{~mm}$ is necessary for the sensor design. From our initial study of the fiber displacement effect using a single axis translational stage (Thorlabs), a poor light reception is produced for a fiber gap of more than $3 \mathrm{~mm}$, which is corresponding to the light intensity estimation in Figure 2. The emitting fiber was fixed inside the plastic tube using a heat gun glue, while the receiving fiber cable was purposely allowed to move freely inside the tube. This arrangement is important to ensure the produced light attenuation is mainly from the fiber movement in longitudinal direction, but not due to light loss from fiber offset in lateral and angular directions. For the experimental work presented in this paper, the applied fiber was Mitsubishi Rayon GHV4001. This is a multimode plastic optical fibre (PMMA) which has a core/cladding diameter of $1 \mathrm{~mm}$, numerical aperture at 0.5 and with step index profile.

The overall sensor schematic is shown in Figure 3.
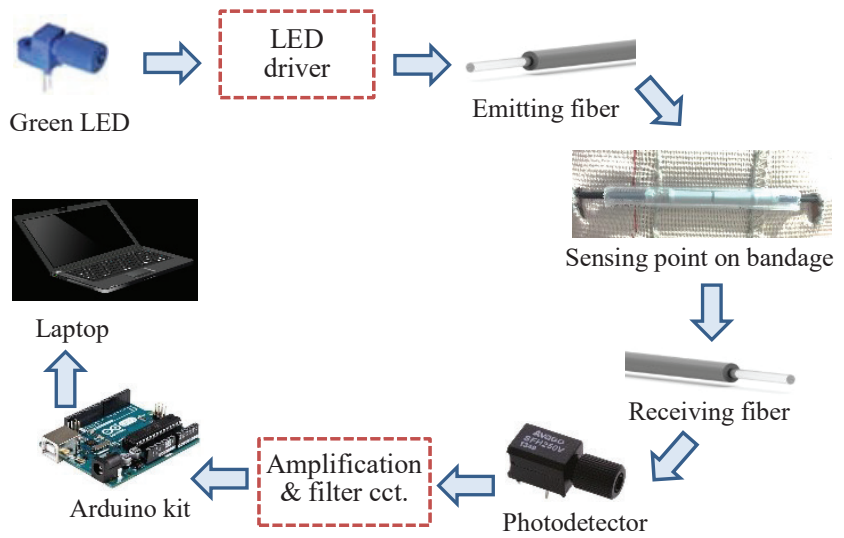

Fig. 3. Fiber optic respiration sensor schematic.
The respiration sensor proposed in this paper is based on the theoretical study and parameter calculation of light intensity variation with respect to different fiber gap as illustrated in Figure 2. In terms of the sensor preparation, the fiber cable was cut using a hot knife at a predetermined length. Then, the jacket of the fiber was removed with a length of $1 \mathrm{~cm}$ at each fiber end tip. The fiber end tips were then polished using a sand paper with different grids before being inserted into a plastic optical fiber (POF)-type v-pin connector to ensure good light acceptance into the fiber. The connector has $1.0 \mathrm{~mm}$ and $3.2 \mathrm{~mm}$ inner and outer diameter, respectively. Both emitting and receiving fibers were held together using a plastic tube to allow the fiber to displace in longitudinal direction with minimum lateral offset.

The bandage (with the optical sensor on it), was then placed encircled a large plastic container as shown in Figure 4 to represent the respiration process in laboratory scale test rig.

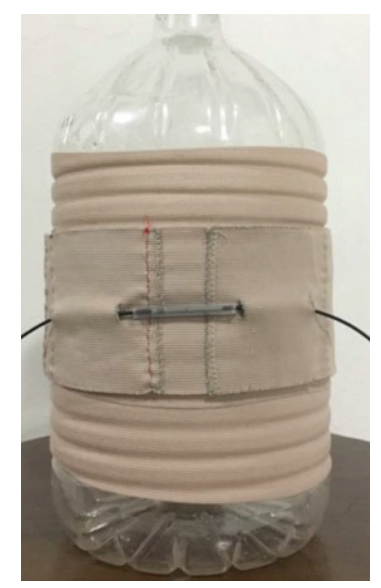

Fig. 4. Respiration sensor placement on a circle object.

\section{Results and analysis}

In the experimental study, the output of the optical sensor is the light intensity detected by a photodetector (with embedded lens), which has a spectral range between 400 and $1100 \mathrm{~nm}$. The optical sensor is supplied by a high brightness green LED with a peak wavelength of $530 \mathrm{~nm}$. The maximum detected voltage from the designed circuit is only at $50 \mathrm{mV}$, which is too small to be displayed on a standard desktop monitor. In order to amplify the signal, a signal conditioning circuit with an amplification gain of 100 is fabricated and applied before the amplified signal is inserted into the Arduino kit analogue pin.

The output responses from this study are shown in Figure 5. To study the capability of the sensor arrangement to maintain its initial fiber gap, the movement of the fiber gap is tested at two different time responses (e.g. fast and slow movement). After multiple trials, the results show that, the respiration sensor is able to return its initial position after being stretched for several times. Moreover, the bandage can also be attached to different body sizes without changing the initial fiber gap because the receiving fiber position on the bandage 
can be adjusted by altering related segment of the bandage.

In the current stage of this study, the sensor has yet to be tested on any human volunteers. Nevertheless, it will be necessary in the future to carry out such experiments to prove the performance and reliability of the proposed optical sensor technique. Another concern is that, although the use of intensity modulation technique, i.e. fiber longitudinal displacement is not considered as advanced sensing methods such as fiber grating and fiber interferometry counterparts, the implementation of this method is considered new for this particular health monitoring application.

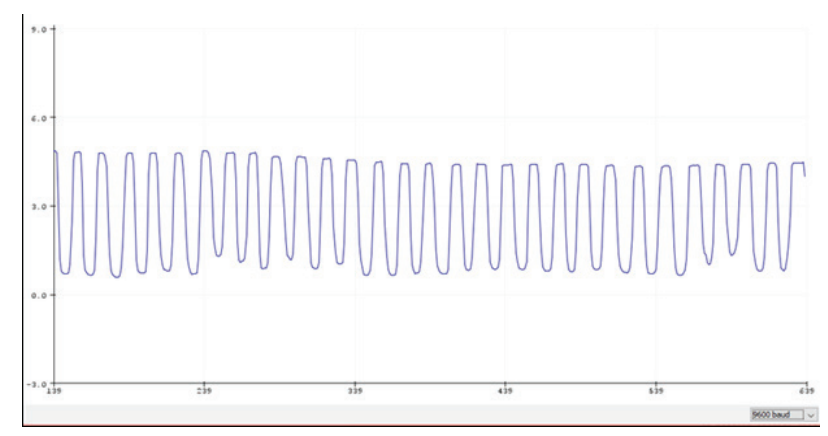

(a) fast response ( $3 \mathrm{sec}$. per cycle)

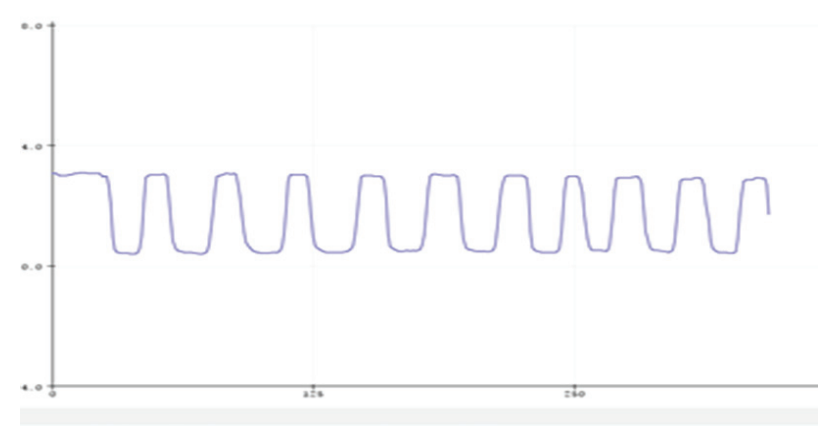

(b) slow response (8 sec. per cycle)

Fig. 5. Output response of the optical sensor during the natural and stretch conditions to represent the chest movement

As a conclusion, the development of a respiration monitoring sensor using plastic optical fiber based on intensity modulation technique has been presented. The inhalation and exhalation process is detected from the longitudinal fiber displacement, due to chest movement where the sensor is located via a stretchable bandage. The fiber gap for sensor assembly was obtained from a theoretical estimation involving different parameters of the applied fiber. The experimental study of the sensor showed a consistent output level during idle and stretched condition.
The authors wish to thank Universiti Malaysia Pahang for the financial support on the research activities via internal research grant RDU150364.

\section{References}

1. C.R. Merritt, H. Troy Nagle, E. Grant, IEEE Sensors Journal. 9, 1, pp. 71-78 (2004)

2. X. Yanming, L. Changzhi, L. Jenshan, IEEE Sensors Journal. 7, 7, pp. 1042-1043 (2007)

3. K.S. Tan, R. Saatchi, H. Elphick, D. Burke, Proc. of the Int Symp. on Communication Systems Networks and Digital Signal Processing, 7, pp. 770-774 (2010)

4. W.J. Yoo, K.W. Jang, J.K. Seo, J.Y. Heo, J.S. Moon, J.H. Jun, J.Y. Park, B. Lee, Optical Review. 18, 1, pp. 132-138 (2011)

5. A. Grillet, D. Kinet, J. Witt, M. Schukar, K. Krebber, F. Pirotte, A. Depre, IEEE Sensors Journal. 8, 7, pp. 1215-1222 (2008)

6. O. Ziemann, J. Krauser, P. E. Zamzow, W. Daum, Optical Short Range Transmission Systems. 2nd ed., Springer, Berlin, Germany, pp. 259-268 (2008)

7. H. Tsuchiya, H. Nakagome, N. Shimizu, S. Ohara, Applied Optics. 16, 5, pp. 1323-1331 (1977) 